CENTRO UNIVERSITÁRIO INTERNACIONALDE CURITIBA - UNINTER

MARIANA PARREIRA LOPES

ABSENTEÍSMO NA EQUIPE DE ENFERMAGEM

SETE LAGOAS

2012

MARIANA PARREIRA LOPES 


\section{ABSENTEÍSMO NA EQUIPE DE ENFERMAGEM}

Trabalho apresentado ao curso de Enfermagem do Trabalho da Faculdade Internacional de Curitiba - FACINTER

Prof(a): Karla Sell Schneider 
SUMÁRIO

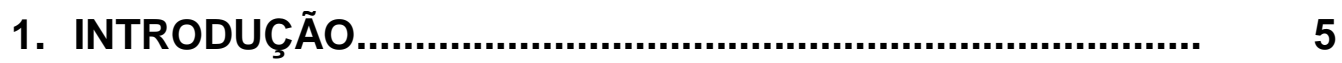

2. DESENVOLVIMENTO....................................................... 6

2.1. Absenteísmo na área de enfermagem.............................. 8

2.2. As causas do absenteísmo.............................................. 11

2.3. Como lidar com o problema............................................ 12

3. Conclusão........................................................................ 13

4. Referências............................................................................ 15 


\section{ABSENTEÍSMO NA EQUIPE DE ENFERMAGEM}

Autor (a): ${ }^{1}$ Mariana Parreira Lopes formada em Enfermagem pela Universidade José do Rosário Vellano - Unifenas BH.

Orientador (a): ${ }^{2}$ Karla Sell Schneider Enfermeira (Unioeste - Universidade estadual do Oeste do Paraná), Especialista em Enfermagem do trabalho (CBES - Colégio Brasileiro de Estudos Sistêmicos) e Estratégia Saúde da Família (UNIPAR - Universidade Paranaense).

\section{RESUMO}

O absenteísmo dos profissionais de enfermagem é um problema complexo para as organizações de saúde, constituindo-se um indicador que necessita ser monitorado. O absenteísmo, genericamente se refere à ausência do trabalhador no local de trabalho. Dentre os denominados fatores humanos no processo de trabalho, que incluem as chamadas doenças ocupacionais e a rotatividade, o absenteísmo se situa entre os efeitos mais danosos ao processo de trabalho, ao suporte social do trabalhador. O absenteísmo se caracteriza, nesse sentido, como tendo um duplo efeito: do ponto de vista do trabalhador, a possibilidade de desconto no salário, de demissão ou de outros problemas correlatos; do ponto de vista da organização do trabalho, a dificuldade de realização do trabalho previsto e os prejuízos por ventura decorrentes. Este trabalho tem o objetivo de demonstrar os danos causados pela falta dos trabalhadores e o que esta falta com o passar do tempo pode trazer para 0 contratante. A queda na produtividade e nos lucros de qualquer instituição é afetada por este motivo. Sendo os profissionais da área de saúde pessoas que lidam diariamente e diretamente com pessoas com saúde debilitada as instituições devem analisar todos os aspectos que influenciam na produtividade do seu empregado, para que o mesmo possa este possa produzir sempre mais e melhor.

Palavras-chave: Absenteísmo; Doença; Enfermagem; Saúde; Trabalhadores 


\section{Introdução}

O consumismo, o individualismo, a competitividade e a agressividade do mercado atual de trabalho são algumas das características do mundo capitalista que o trabalhador vivência, podendo interferir diretamente na sua saúde, gerando prejuízo para o profissional. O ambiente competitivo exige elevado dinamismo, grande esforço físico e psicológico, ultrapassando, muitas vezes, o limite da capacidade do trabalhador. Porém, para manter-se nesse mercado e garantir o emprego, o trabalhador se submete às exigências da instituição na qual se encontra. Assim, as reivindicações por produtividade e qualidade são intensas e crescentes, como também, são elevadas a inconstância e incerteza do emprego.

Reportando-se a definição da palavra absenteísmo define-a como ausência habitual do emprego (FERREIRA, 1986). O absenteísmo, absentismo ou ausentismo é uma expressão utilizada para designar a falta do empregado ao trabalho. Isto é, a soma dos períodos em que os empregados de

determinada organização se encontram ausentes do trabalho, não sendo a ausência motivada por desemprego, doença prolongada ou licença legal (CHIAVENATO, 1994, p.119).

O absenteísmo é hoje uma realidade, tem presença consolidada no mercado de trabalho. Já existem hoje instituições que fazem ao máximo para agradar os seus trabalhadores para que este produza mais e com isso seus lucros aumentem.

No Brasil não existem pesquisas feitas para uma análise mais profunda. Mas existem diversos artigos citando o absenteísmo na área de saúde e principalmente na equipe de enfermagem. Mas será porque estes profissionais são mais afetados? Será que os profissionais da área de saúde têm suporte para atender os pacientes adequadamente? Será que o problema está na parte administrativa, os supervisores e diretores, escutam seus trabalhadores para tentar solucionar o problema? Muitas perguntas vão ser abordadas nesta pesquisa. 
Em virtude das condições desfavoráveis presentes no ambiente de trabalho, os trabalhadores acabam por buscar meios de compensar 0 sofrimento, tornando-se funcionários resistentes e adotando posturas defensivas. Tornam-se indiferentes ao processo laboral, optando pela fuga ao trabalho, que pode ocorrer por meio de atestados, licenças médicas ou simplesmente por faltas injustificadas que afetam os próprios trabalhadores, bem como as organizações, comprometendo os resultados finais dos serviços (ALVES, 1996).

A pesquisa tem como objetivo abordar o absenteísmo na área da saúde. Mostrando os possíveis motivos que levam o profissional se abster do trabalho. Além de mostrar o que é necessário para que este trabalhador fique satisfeito com seu ambiente de trabalho e com isso desempenhe bem o seu trabalho. A pesquisa consisitirá em pesquisa bibliográfica. A opção se justifica pelo fato de demonstrar quais os possíveis motivos do absenteísmo e alguns prejuízos para as empresas. Para que o resultado final desse trabalho seja relevante.

O estudo foi realizado por meio de busca online das produções científicas nacionais sobre o absenteísmo dos profissionais de enfermagem. A captura dessas produções foi processada por meio da Biblioteca Virtual em Saúde (BVS), sendo utilizadas as bases de dados: Literatura Latino-Americana e do Caribe em Ciências da Saúde (LILACS), Scientific Electronic Library Online (SciELO), e o levantamento no portal da Coordenação de Aperfeiçoamento de Pessoal de Ensino Superior (CAPES). Estabeleceram-se como critérios para a seleção da amostra: artigos publicados no Brasil e que apresenta de maneira explicita os descritores absenteísmo e enfermagem no resumo. Os descritores utilizados na busca foram "absenteísmo" e "enfermagem".

\section{Desenvolvimento}

O trabalho é importante na vida das pessoas, seja como fator de crescimento e realização pessoal ou, em uma visão menos idealizada, como meio de sobrevivência. Por suas determinações históricas e econômicas, o 
trabalho pode ser compreendido como organizador da vida social, embora estabeleça caminhos para a dominação cultural, social e econômica e para a submissão do trabalhador ao capital. Para um conjunto de pessoas é reservado o direito de pensar e planejar; para outros cabe apenas a execução, sendo esses, via de regra, pertencentes à base da pirâmide social (ALVES, 1996).

A análise da relação capital/trabalho revela contradições, pois o mesmo trabalho que dignifica, confere status e reconhecimento ao ser humano, pode ser também fonte de sofrimento, de desequilíbrio físico e mental, de dor e frustração. Tudo isso se agrava com o estabelecimento do modelo capitalista e a exacerbação dos ditames da administração científica (REZENDE, 2003). Nesse contexto, as condições de trabalho tornam-se insalubres, a ponto de agredir a dignidade dos trabalhadores, caracterizando uma prática laboral incompatível com a qualidade de vida (ALVES, 2001).

Ao abordar o trabalho em instituições de saúde e relacioná-lo às condições ambientais, políticas e gerenciais, pode-se analisar a gênese de uma série de agravantes da integridade e da saúde do trabalhador. Assim, deve-se levar em conta a peculiaridade do ambiente hospitalar, onde os trabalhadores estão permanentemente em contato com o sofrimento e a morte (LUNARDI, 1997). Considera-se ainda que a maioria das instituições de saúde possua estrutura político-administrativa pouco flexível, dificultando a autonomia dos trabalhadores. Mesmo com a utilização máxima de suas faculdades intelectuais e psicoafetivas de aprendizagem, os trabalhadores não conseguem adaptar-se à estrutura das instituições (DEJOURS, 2000).

Segundo QUICK \& LAPERTOSA (1982), o absenteísmo é dividido em absenteísmo voluntário (ausência no trabalho por razões particulares não justificadas por doença); absenteísmo por doença (inclui todas as ausências por doença ou por procedimento médico, excetuam-se os infortúnios profissionais); absenteísmo por patologia profissional (ausências por acidente de trabalho ou doença profissional); absenteísmo legal (faltas no serviço amparado por leis, tais como: gestação, doação de sangue e serviço militar) e absenteísmo compulsório (impedimento ao trabalho devido à suspensão imposta pelo patrão, por prisão ou outro impedimento que não permita o trabalhador chegar ao local de trabalho). 
Um aspecto importante a ser considerado é que as causas do absenteísmo nem sempre estão no trabalhador, mas na empresa, enquanto organização e supervisão deficientes, através da repetitividade de tarefas, da desmotivação e desestímulo, das condições desfavoráveis de ambiente e de trabalho, da precária integração entre os empregados e a organização e dos impactos psicológicos de uma direção deficiente, que não visa uma política prevencionista e humanista (ALEXANDRE, 1987; COUTO, 1987; CHIAVENATO, 1994; ALVES, 1995).

No Brasil não existe nenhuma pesquisa específica ou estudos oficiais quanto ao prejuízo financeiro do país em relação ao absenteísmo. Seja ele por à incapacidade para o trabalho por doença, acidente ou lesão. Isto nos leva a crer que ainda não é prioridade do governo (NOGUEIRA E AZEVEDO, 1996).

O número insuficiente de recursos humanos pode contribuir para elevar o índice de absenteísmo, como consequência da sobrecarga e insatisfação dos trabalhadores, desencadeando a queda da qualidade do cuidado prestado ao homem (ALVES, 1995).

\subsection{Absenteísmo na área de enfermagem}

No contexto hospitalar, a enfermagem constitui-se na maior força de trabalho, e suas atividades são frequentemente marcadas por divisão fragmentada de tarefas, rígida estrutura hierárquica para o cumprimento de rotinas, normas e regulamentos, dimensionamento qualitativo e quantitativo insuficiente de pessoal, situação de exercício profissional que tem repercutido em elevado absenteísmo e afastamentos por doenças.

Pouco se sabe sobre como o trabalhador se sente frente ao trabalho em si, suas relações com colegas, chefias, sistemas de salários e benefícios. Certamente a compatibilização destas expectativas com as necessidades organizacionais, torna-se um desafio diante de obstáculos que se apresentam como longas jornadas de trabalho, condições de insalubridade do ambiente de trabalho; baixa remuneração; duplo emprego e tensão emocional. Estes obstáculos podem desencadear o absenteísmo. Esta situação é preocupante, 
uma vez que desorganiza o serviço, gera insatisfação e sobrecarrega os trabalhadores presentes, diminuindo a qualidade da atividade realizada (SILVA, 2006).

Muito se tem escrito sobre as condições inadequadas de trabalho vigentes em grande parte dos ambientes hospitalares, que expõem seus trabalhadores a riscos de ordem biológica, física, química, psicológica e social. A instituição hospitalar caracteriza-se como local de aglutinação de pacientes/cliente acometido por diferentes problemas de saúde. Dentre estes se destaca problemas administrativos, prejuízos à saúde física e mental dos trabalhadores, quando expostos a prolongadas jornadas de trabalho; ritmo acelerado de produção, por excesso de tarefas; automação por realização de ações repetidas com parcelamento de tarefas e remuneração baixa, em relação à responsabilidade e complexidade das tarefas. Além de refletir conflitos nas relações de trabalho, interfere na satisfação do trabalhador, eleva os custos e contribui para o declínio da qualidade de assistência, afetando, a organização, trabalhadores e clientes. O absenteísmo pode estar diretamente relacionado às condições de trabalho, refletindo na qualidade e produtividade laboral e na vida do trabalhador de enfermagem.

Considerando a enfermagem como profissão onde a maior parte dos profissionais são mulheres e as questões socioculturais da inserção da mulher no mercado de trabalho, torna-se relevante lembrar que muitas delas têm dupla jornada de trabalho (BARBOZA; SOLER, 2003). Pensando nisso há também necessidade de acompanhar mais atentamente o trabalho desenvolvido pelos servidores mais velhos a fim de verificar se o absenteísmo-doença está relacionado à sobrecarga de trabalho biológico.

As instituições de saúde, a organização do trabalho da equipe de enfermagem é essencial para o atendimento adequado e de qualidade ao cliente/paciente. Analisando o contexto do processo de trabalho desses profissionais, onde predominam as atividades complexas, tarefas que requerem habilidades e conhecimentos técnico-científicos devido aos cuidados que demandam atenção permanente e maior carga de trabalho da equipe de enfermagem, há necessidade de garantir número adequado de trabalhadores 
para assegurar a qualidade da assistência de enfermagem durante as 24 horas do dia.

Segundo REIS (1986), o absenteísmo apresenta-se como um obstáculo para as chefias de enfermagem manter a qualidade da assistência, acrescido às limitações no desempenho de suas funções frente aos trabalhos que dispensam um esforço físico maior.

Para ROBAZZI et al. (1990), as ausências de funcinários de enfermagem desorganizam o trabalho de equipe e vão alterar a qualidade e quantidade do atendimento prestado ao paciente, desencadeando problemas também aos enfermeiros que ocupam cargos de chefia, pois têm sob sua responsabilidade a resolução de todos os problemas administrativos que lá acontecem. Sendo assim os supervisores tem que alterar toda a escala do dia já que os pacientes internados nas instituições precisam de assistência e isso não pode ser adiado, visto que sua recuperação pode ser prejudicada. Esta ausência de um membro da equipe causa uma sobrecarga nos outros trabalhadores além de uma perturbação para que seja realizado todos os trabalhos. Reduz a produção, aumenta o custo operacional, dificulta a substituição de trabalhadores diretamente ligados à assistência, caindo sensivelmente a qualidade do cuidado prestado.

O aumento das faltas no trabalho devido à licença-saúde na enfermagem pode ser decorrente do tipo de trabalho e da insalubridade do ambiente ocupacional (SILVA; MARZIALE, 2006). O não reconhecimento também é um dos fatores pelos quais os profissionais dessa área podem apresentar uma insatisfação no trabalho e que leva a algumas doenças psicológicas ocupacionais. Apesar de trabalhar na busca da saúde para os outros, a equipe de enfermagem tem se excluído do merecimento dessa atenção. Uma atenção que lhe é devida de direito, e da qual depende para continuar trabalhando em condições de dar a contribuição dela esperada (BULHÕES, 1998).

O absenteísmo por doença é considerado o principal motivo das faltas imprevistas em unidades hospitalares (SILVA; MARZIALE, 2006; INOUE et al, 2008). Os afastamentos motivados por doença são frequentes e trazem 
prejuízos financeiros à instituição hospitalar, compromete a qualidade do cuidado prestado aos pacientes e sobrecarrega para a equipe de enfermagem.

As condições de trabalho de enfermagem implicam em longas jornadas, no trabalho em turnos desgastantes (matutino, vespertino e noturno, domingos e feriados), nos rodízios, em multiplicidade de funções, repetitividade e monotonia, intensividade e ritmo excessivo de trabalho, ansiedade, esforços físicos, posições incômodas, na separação do trabalho intelectual e manual, no controle das chefias, desencadeando acidentes e doenças (ALVES, 1995). Muitas vezes, trabalhadores com problemas de saúde querem físicos e/ou mentais, segundo MOURA (1992) transferem seus problemas para o trabalho, os quais podem ocasionar atrasos, faltas, descuido com o material e queda na qualidade do trabalho executado.

Os riscos ocupacionais variam de acordo com as atividades exercidas e o meio ambiente. A sobrecarga de risco pode desencadear prejuízo para a saúde do trabalhador, provocando o absenteísmo.

\subsection{As causas do absenteísmo}

O absenteísmo pode ser atribuído a causas conhecidas e a causas ignoradas. Dentre as conhecidas, estão todas as amparadas por lei e que são, por isso mesmo, justificada ao empregador, solicitando-Ihe a permissão de ausência. É o caso de férias, casamentos, nascimentos, óbitos e mudanças de domicilio. As ignoradas são justificadas geralmente por problemas de saúde do trabalhador, e ou de seus dependentes ou de fatores aleatórios dos mais diversos

As principais causas do absenteísmo são consideradas: doença, razões diversas de caráter familiar, atrasos involuntários ou por motivos de força maior, faltas voluntárias por motivos pessoais, dificuldades e problemas financeiros, problemas de transporte, baixa motivação para trabalhar, supervisão precária da chefia, políticas inadequadas da organização (SOUTO, 1980). 


\subsection{Como lidar com o problema?}

Quando falamos de política de absenteísmo orientada para a prevenção das causas que the estão subjacentes, estamos nos referindo às medidas concretas que a empresa toma e que são basicamente as seguintes:

A) Medidas processuais, administrativas ou disciplinares, dificultando ou facilitando o comportamento de ausência (por ex., precarização do emprego, perda de vencimento e / ou de prêmios de assiduidade, complemento de subsídio de doença pago ou não pela empresa, exames de alta feita pelo médico do trabalho);

B) Medidas preventivas orientadas para o indivíduo e o reforço da sua capacidade de trabalho (por ex., exames médicos periódicos, vacinação, formação, equipamento de proteção individual contra os riscos profissionais, educação para a saúde);

C) Medidas preventivas orientadas para o ambiente de trabalho físico e psicossocial, de modo a neutralizar, reduzir ou minimizar a discrepância entre as exigências impostas pelo trabalho e a capacidade de resposta do indivíduo (por ex., criação sustentada de um ambiente de trabalho saudável, seguro e produtivo, participação na organização do trabalho e na gestão, participação no sistema de gestão de pessoas);

D) E, finalmente, medidas reintegrativas, ou seja, orientadas para a reintegração e reabilitação no local de trabalho, facilitando e apressando 0 retorno ao trabalho (por ex., suporte social do grupo de trabalho, política de incentivos, serviços ou programas de reabilitação no local de trabalho).

\section{Conclusão}

Constata-se que o tema absenteísmo ainda não ocupa uma posição estratégica dentro das organizações e também nas esferas políticas, ou melhor, dizendo governamentais, devido à carência de pesquisa dentro desta área da saúde. 
O processo de trabalho dos profissionais de enfermagem tem acarretado inúmeras consequências como os acidentes e as doenças relacionadas ao trabalho. Neste contexto, o absenteísmo doença tem merecido a atenção, pelo alto índice de ocorrência.

Concluímos que os índices de absenteísmo doença entre os trabalhadores de enfermagem nas instituições de saúde apesar de elevadas não se sabe precisar a quantidade exata. Sugerindo então a necessidade de estudos em cada local de trabalho, buscando detectar problemas causais específicos de cada setor e planejar soluções. Há também a necessidade de criação de um banco de dados para aperfeiçoar o registro das faltas, afim de facilitar seu controle e possibilitar futuras pesquisas.

Para compreender os modelos desenvolvidos atualmente na condução das pessoas nas diversas organizações precisa-se conhecer um pouco sobre a complexidade das organizações e os modelos adotados na administração das pessoas no processo produtivo.

Primeiramente deve-se entender que quanto mais evoluída a sociedade mais demandas são criadas, em função disto mais empresas são aperfeiçoadas e mais complexas são os processos desenvolvidos.

A gestão de pessoas vem para auxiliar a coordenação dos esforços das pessoas que trabalham em uma organização e que tem como objetivo tornar real a missão organizacional bem como promover a realização pessoal. Nenhuma empresa sobrevive sem esta sinergia.

Atualmente percebe-se um esforço constante e intenso de profissionais da área de gestão de pessoas buscando uma mudança radical de postura. As pessoas não precisam ser administradas e sim devem ser consideradas parceiras das organizações, cujos objetivos tanto pessoais quanto organizacionais caminham em uma mesma direção. Os profissionais são respeitados e valorizados porque são sujeitos ativos e proativos, que estruturam estratégias e as colocam em prática potencializando as empresas no mercado de atuação.

Nesta nova percepção de relação de trabalho, as pessoas são parceiras das organizações o que implica em uma troca justa onde os colaboradores 
entram com esforço, dedicação, produtividade e colhem retornos interessantes e significativos para suas vidas, ou seja, a particularidade de cada um deve ser considerada. Cada pessoa, percebida como um microambientes tem seus objetivos específicos e buscam na organização a satisfação destes, portanto 0 que é significativo para um pode não ser para o outro e administrar com as pessoas é levar em consideração esta especificidade.

Espera-se também que criem um banco de talentos para que nele existam profissionais que possam substituir os faltosos. Seria como contratar funcionários temporários por efetivos, principalmente na categoria profissional de enfermagem de nível médio, haja vista que o trabalho de assistência de enfermagem é feita na maioria das vezes por esses profissionais, e os pacientes não podem esperar pelo atendimento para que não tenha um comprometimento da sua reabilitação.

Sugere-se que outros estudos sejam realizados sobre o tempo de trabalho nas instituições de saúde, o modelo organizacional e a motivação para o trabalho, como causas do absenteísmo relacionado ao adoecimento, a fim de se atenuar seus efeitos negativos na qualidade da assistência de enfermagem. Sugere-se, também, a criação de um banco de dados, a ser instalado nos hospitais, para aperfeiçoar o registro das faltas, para acompanhamento e avaliação e permitir outras pesquisas. 
Referências

ALEXANDRE, N.M.C. Avaliação de determinados aspectos ergonômicos no transporte de pacientes. Ribeirão Preto, 1987. Dissertação (Mestrado) Escola de Enfermagem de Ribeirão Preto, Universidade de São Paulo.

ALVES, A.R.A. Avaliação diagnostica dos índices de absenteísmo da equipe de Enfermagem de um Hospital de Ensino. Fortaleza, 1995. 74p.

ALVES, M.. Causa de Absenteísmo entre auxiliares de enfermagem: uma dimensão do sofrimento no trabalho [tese]. São Paulo (SP): Escola de Enfermagem da USP, Universidade de São Paulo; 1996.

ALVES, M, Godoy SCB. Procura pelo serviço de atenção à saúde do trabalhador e absenteísmo - doença em um hospital universitário. Rev Min Enferm 2001; 5(1): 73-81.

BARBOZA, D.B.; SOLER, Z.A.S.G. - Afastamentos do Trabalho na enfermagem: ocorrências com trabalhadores de um hospital de ensino. Rev. Latino-Am Enfermagem v.11, n.2, Ribeirão Preto Mar./Apr, 2003. [citado em: 2 abr 2011]. Disponível em: URL http://www.scielo.br/scielo.php?script=sci arttext\&pid=S0104$\underline{11692003000200006}$

BULHÕES, I. Enfermagem do trabalho. Rio de Janeiro: IDEAS, 1986. v. 2, $463 \mathrm{p}$.

Riscos do trabalho de enfermagem. 2.ed. Rio de Janeiro: Correio Carioca, 1998. 221p. [citado em: 14 mai 2012]. Disponível em: URL http://www.abeneventos.com.br/anais 61cben/files/01817.pdf 
CHIAVENATO, I. Recursos humanos. São Paulo: Atlas, 1985. 377p.

Recursos humanos na empresa. 3. ed. São Paulo: Atlas, 1994. v. 2, 139p.

COSTA, F. M. da; VIEIRA, M. A.; SENA, R. R. de. Absenteísmo relacionado à doenças entre membros da equipe de enfermagem de um hospital escola. Rev. bras. enferm. [online]. 2009, vol.62, n.1, pp. 38-44. ISSN 00347167. http://dx.doi.org/10.1590/S0034-71672009000100006.

COUTO, H. A. Temas de Saúde Ocupacional: coletânea dos cadernos ERGO. 1. ed. Belo Horizonte: ERGO, 1987

DEJOURS, C. A loucura do trabalho: estudo de psicopatologia do trabalho. 5a ed. São Paulo: Cortez-Oboré; 2000.

INOUE, K.C. et al. Absenteísmo-doença da equipe de enfermagem em unidade de terapia intensiva. Rev. bras. enferm. [online]. 2008, vol.61, n.2, pp. 209-214. ISSN 0034-7167. http://dx.doi.org/10.1590/S003471672008000200010 .

PENATTI, I.; ZAGO, J. S.; QUELHAS, O. Absenteísmo: As consequências na gestão de pessoas [citado em: 15 mai 2012]. Disponível em: URL: http://www.aedb.br/seget/artigos06/898 Seget Izidro\%20Penatti.pdf

LUNARDI, W.D.F. Prazer e sofrimento no trabalho: contribuições à organização do processo de trabalho de enfermagem. Rev Bras Enferm 1997; 50(1): 77-92.

MARZIALE, M.H.P. Condições ergonômicas da situação de trabalho do pessoal de enfermagem, em uma unidade de internação hospitalar. 
Ribeirão Preto, 1995. 155p. Tese (Doutorado) - Escola de Enfermagem de Ribeirão Preto, Universidade de São Paulo

MATOS, Mauro Gomes; HENNINGTON, Élida Azevedo; HOEFEL, Ana Lúcia and DIAS-DA-COSTA, Juvenal Soares. Dor lombar em usuários de um plano de saúde: prevalência e fatores associados. Cad. Saúde Pública [online]. $\quad 2008, \quad$ vol.24, $\quad$ n.9, pp. 2115-2122. ISSN 0102311X. http://dx.doi.org/10.1590/S0102-311X2008000900017

MOURA, G.M.S.S.de. 0 estudo da satisfação no trabalho e do clima organizacional como fatores contributivos para o ser saudável no trabalho da enfermagem. Texto Contexto Enfermagem, Florianópolis, v. 2, n. 1, p. 167-179, jul./dez. 1992 [citado em: 14 mai 2012]. Disponível em: URL: http://www.scielo.br/pdf/rlae/v8n5/12366.pdf

NOGUEIRA, D. Pupo, A.ZEVEDO, C. A. B. Absentismo-doença em mulheres. Revista Brasileira de Saúde Ocupacional. São Paulo: Fundacentro, 1996.

QUICK,T.C.: LAPERTOSA , J.B. Analise do absenteísmo em usina siderúrgica. Ver. Brás. Saúde Ocupacional., v.10,n.40 , 1982

REIS, I.N. Doenças ocupacionais: estudo retrospectivo em unidades hospitalares do DF. HFA - Publ. Téc. Cient., v. 1, n. 2, p. 113-122, jul./set. 1986.

REZENDE, MP. Agravos à saúde de auxiliares de enfermagem resultantes da exposição ocupacional aos riscos físicos [dissertação]. Ribeirão Preto: Escola de Enfermagem da USP, Universidade de São Paulo; 2003. 
ROBAZZI, M.L.C.C.; PARACCHINI, S.A. ; GIR, E. ; SANTOS, W.D.F. dos; MORIYA, T.M. Serviço de enfermagem: um estudo sobre os absenteísmos. Rev. Bras. Saúde Ocup., v. 18, n. 69, p. 65-70, jan./fev./mar. 1990.

SANCINETTI, T.R. et al. Taxa de absenteísmo da equipe de enfermagem como indicador de gestão de pessoas. Rev. esc. enferm. USP [online]. 2011, vol.45, n.4, pp. 1007-1012. ISSN 0080-6234. .

T.R. et al. Absenteísmo - doença na equipe de enfermagem: relação com a taxa de ocupação. Rev. esc. enferm. USP [online]. 2009, vol.43, n.spe2, pp. 1277-1283. ISSN 0080-6234.

SILVA, D.M.P.P.; MARZIALE, M.H.P. - Condições de trabalho versus absenteísmo-doença no trabalho de enfermagem. Rev. Ciência, Cuidado e Saúde, Maringá, v.5, Supl., p.166-172, 2006. [citado em: 28 abr 2012]. Disponível em:

http://periodicos.uem.br/ojs/index.php/CiencCuidSaude/article/view/5187/3355

DMPP, Marziale MHP. Absenteísmo de trabalhadores de enfermagem em um hospital universitário. Rev Latino-am Enfermagem 2000 out ; 8(5): 44-51.

SOUTO, D. F. Absenteísmo, preocupações constante das organizações. Temas de Saúde Ocupacional. Eletrobrás. Gridis, 1980 